BULL. AUSTRAL. MATH. SOC.

\title{
DISSIPATIVE OPERATORS AND \\ SERIES INEQUALITIES
}

\section{Herbert A. Gindler and Jerome A, Goldstein}

\begin{abstract}
of concern is the best constant $K$ in the inequality $\|A x\|^{2} \leq K\left\|A^{2} x\right\|\|x\|$ where $A$ generates a strongly continuous contraction semigroup in a Hilbert space. Criteria are obtained for approximate extremal vectors $x$ when $K=2$ ( $K \leq 2$ always holds). By specializing $A+I$ to be a shift operator on a sequence space, very simple proofs of Copson's recent results on series inequalities follow. Inequalities of the above type are also stuaied on $L^{P}$ spaces, and earlier results of the authors and of Holbrook are improved.
\end{abstract}

\section{Introduction}

There is a large literature on norm inequalities involving dissipative operators on Banach spaces. This literature can be traced back to inequalities of Landau, Hardy and Littlewood which take the form (1.1) $\quad\left\{\int_{J}\left|f^{\prime}(x)\right|^{p} d x\right\}^{2 / p} \leq K\left\{\int_{J}\left|f^{\prime \prime}(x)\right|^{p} d x\right\}^{1 / p}\left\{\int_{J}|f(x)|^{p} d x\right\}^{1 / p}$ where $J$ is $[0, \infty)$ or $(-\infty, \infty)$ and $1 \leq p \leq \infty$ (with the usual interpretation for $p=\infty$ ). Recently Copson [2] established some

Received 9 January 1981. J.A. Goldstein was partially supported by an N.S.F. grant. This paper was written while H.A. Gindler was on sabbatical leave from San Diego State University. He would like to thank the faculty and staff of the Department of Mathematics of Tulane University for their gracious hospitality during his stay. 
inequalities for infinite series based on an analogy with the case $p=2$ in (1.1). One of our purposes is to show that Copson's results follow easily from certain operator theoretic versions of (1.1).

Our attempt to generalize [2] led quite naturally to questions concerning the existence of extremals and approximate extremals in the operator theoretic versions of the case $p=2$ of (1.1). This led to results which can be considered as extensions of and were motivated by the works of Kato [6] and of Kwong and ZettI [7], [8].

In the final section we establish some inequalities involving dissipative operators on $L^{P}$ spaces. These include series inequalities (in $\imath^{p}$ norms) and other inequalities as well. These results are obtained using techniques we introauced in [3]. One of the theorems in this section was motivated by the work of Holbrook [4].

\section{Approximate extremals in Hilbert space}

Let $A$ be a linear operator on its domain $D(A) \subset X$ to $X$, where $X$ is a real or complex Banach space. As in [3] let

$$
C(X ; A)=\inf \left\{k:\|A x\|^{2} \leq k\left\|A^{2} x\right\|\|x\| \text { for all } x \in D\left(A^{2}\right)\right\} .
$$

PROPOSITION 2.1. Let $L \neq I$ be a contraction on $x$ (that is, $\|L\| \leq 1$, , and let $A=L-I$. Then $A$ is m-dissipative and $1 \leq C(X ; A) \leq 4$. Moreover, if $X$ is a Hilbert space, then $C(X ; A) \leq 2$, and $C(X ; A)=1$ if $A$ is normal.

Proof. If $L$ is a contraction and $t>0$, then

$$
\left\|e^{t A}\right\|=e^{-t}\left\|e^{t L}\right\| \leq e^{-t} e^{t\|L\|}=1 \text {, }
$$

whence the semigroup $\left\{e^{t A}: t \geq 0\right\}$ generated by $A$ is contractive, and so $A$ is m-dissipative [10]. The inequality $C(X ; A) \leq 4$ for $m$-dissipative operators $A$ was proved by Kallman and Rota [5]. Kato [6] showed that $C(X ; A) \leq 2$ holds if $X$ is a Hilbert space. If $A$ (or $L$ ) is normal, then

$$
\|A x\|^{2}=(A x, A x\rangle=\left\langle A^{*} A x, x\right\rangle \leq\left\|A^{*} A x\right\|\|x\|=\left\|A^{2} x\right\|\|x\|
$$

because $\left\|A^{*} y\right\|=\|A y\|$ by normality. Thus $C(X ; A) \leq 1$. This was noted 
earlier in [3], [9]. It only remains to show that $C(X ; A) \geq 1$ in all cases. Since $L \neq I$ is equivalent to $A \neq 0$, choose unit vectors $x_{n} \in X$ with $\lim _{n \rightarrow \infty}\left\|A x_{n}\right\|=\|A\|$. From

$$
\|A\|^{2}=\lim _{n \rightarrow \infty}\left\|A x_{n}\right\|^{2} \leq C(X ; A) \underset{n \rightarrow \infty}{\lim _{n \rightarrow \infty}}\left\|A^{2} x_{n}\right\| \leq C(X ; A)\|A\|^{2}
$$

it follows that $C(X ; A) \geq 1$.

of course, $C(X ; A)=0$ if and only if $A=0$ if and only if $L=I$, which is trivial.

Now let $A$ be any operator with $C(X ; A)$ finite. An extremal for $A$ is a unit vector $x$ in $O\left(A^{2}\right)$ such that $A^{2} x \neq 0$ and

$$
\|A x\|^{2}=C(X ; A)\left\|A^{2} x\right\| \text {. }
$$

An approximate extremal sequence for $A$ is a sequence $\left\{x_{n}\right\}$ of unit vectors in $D\left(A^{2}\right)$ such that $A^{2} x_{n} \neq 0$ and

$$
\lim _{n \rightarrow \infty}\left\|A x_{n}\right\|^{2}\left\|A^{2} x_{n}\right\|^{-1}=C(X ; A) .
$$

THEOREM 2.2. Let $A$ be an m-dissipative operator on a Hilbert space $H$. Then:

(i) $C(H ; A) \leq 2$;

(ii) $C(H ; A)=2$ and there is an extremat for $A$ if and only if there is a unit vector $x$ in $D(A)$ and a positive constant $\lambda$ such that

$$
A^{2} x+\lambda A x+\lambda^{2} x=0
$$

and

$$
\operatorname{Re}\left(A^{2} x, x\right)=0
$$

where $(\cdot, \cdot)$ denotes the inner product on $H$ and $\mathrm{Re}$ denotes the real part of a complex number;

(iii) if there is a sequence of unit vectors $\left\{x_{n}\right\}$ in $D\left(A^{2}\right)$ 
and a positive constant $\lambda$ such that

(2.3) $A^{2} x_{n} \rightarrow 0, A^{2} x_{n}+\lambda A x_{n}+\lambda^{2} x_{n} \rightarrow 0$,

$$
\text { and } \operatorname{Re}\left\langle A^{2} x_{n}, x_{n}\right\rangle \rightarrow 0 \text { as } n \rightarrow \infty \text {, }
$$

then $C(H ; A)=2$ and $\left\{x_{n}\right\}$ is an approximate extremal

sequence for $A$;

(iv) conversely, if $A$ and $A^{-1}$ are bounded and if

$C(H ; A)=2$, then there is a sequence of unit vectors

$\left\{x_{n}\right\}$ in $D\left(A^{2}\right)$ and $a \quad \lambda>0$ satisfying (2.3).

Proof. Parts (i) and (ii) are due to Kato [6], while (iii) and (iv) are new. Our proof of (iii), which is based on the work of Kwong and Zet+1 [7], will prove (i) and (ii) as well. To begin with, let $\mu>0$ and define

$$
P_{\mu}=A^{2}+\mu A+\mu^{2} I
$$

For $x \in D\left(A^{2}\right)$ define $\alpha=\alpha(\mu, x)$ by

$$
\alpha=2 \operatorname{Re}\langle A(A x+\mu x), \mu(A x+\mu x)\rangle .
$$

Clearly $\alpha \leq 0$ since $A$ is dissipative. Also, an examination of $\left\langle P_{\mu} x, P_{\mu} x\right\rangle$ expanded by linearity yields the identity

$$
\alpha=\left\|P_{\mu} x\right\|^{2}-\left\|A^{2} x\right\|^{2}-\mu^{4}\|x\|^{2}+\mu^{2}\|A x\|^{2} .
$$

If $A^{2} x=0$ then $A x=0$ by dissipativity. If $A x \neq 0$ we set $\mu=\left\{\left\|A^{2} x\right\| /\|x\|\right\}^{\frac{7}{2}}$ in (2.4). We deduce, after dividing by $\mu^{2}$,

$$
\|A x\|^{2}-\alpha\|x\|\left\|A^{2} x\right\|^{-1}+\left\|P_{\mu} x\right\|^{2}\|x\|\left\|A^{2} x\right\|^{-1}=2\left\|A^{2} x\right\|\|x\| .
$$

Since $\alpha \leq 0,(2.5)$ implies that $C(H ; A) \leq 2$. Moreover, $C(H ; A)=2$ and a unit vector $x$ is an extremal for $A$ if and only if $\alpha=0$ and $P_{\mu} x=0$ in (2.5). But $P_{\mu} x=0$ is equivalent to (2.1) and $\alpha=0$ reduces to (2.2). Thus (i) and (ii) are proved.

Using (2.5) again, $C(H ; A)=2$ if and only if there is a sequence 
$\left\{x_{n}\right\}$ of unit vectors in $D\left(A^{2}\right)$ such that $A^{2} x_{n} \neq 0$ and

$$
\lim _{n \rightarrow \infty}\left(\left\|P_{\mu_{n}} x_{n}\right\|^{2}-\alpha_{n}\right)\left\|A^{2} x_{n}\right\|^{-1}=0
$$

where $\mu_{n}=\left\|A^{2} x_{n}\right\|^{\frac{1}{2}}$ and $\alpha_{n}=\alpha\left(\mu_{n}, x_{n}\right)$. (This makes

$\left.\left\|A x_{n}\right\|^{2}\left\|A^{2} x_{n}\right\|^{-1}+2.\right)$ Unfortunately this condition, which is both

necessary and sufficient for $\left\{x_{n}\right\}$ to be an approximate extremal sequence for $A$, is rather cumbersome. Thus we turn to the simpler condition of (iii).

The hypothesis of (iii) implies, by (2.5),

$$
\left\|A x_{n}\right\|^{2}+\left(\left\|P \lambda^{x_{n}}\right\|^{2}-\alpha_{n}\right)\left\|A^{2} x_{n}\right\|^{-1}=2\left\|A^{2} x_{n}\right\|
$$

where $\alpha_{n}=\alpha\left(\lambda, x_{n}\right) \leq 0$. By taking a subsequence if necessary we may assume $\left\|A^{2} x_{n}\right\|$ is bounded away from zero. By hypothesis, $\lim _{n \rightarrow \infty} P_{\lambda} x_{n}=0$ and

$$
\begin{aligned}
\alpha_{n} & =2 \operatorname{Re}\left\langle\left(A^{2}+\lambda A\right) x_{n}, \lambda\left(A x_{n}+\lambda x_{n}\right)\right\rangle \\
& =2 \operatorname{Re}\left\langle-\lambda^{2} x_{n},-A^{2} x_{n}\right\rangle+o(1) \text { since } P_{\lambda^{2} x_{n}} \rightarrow 0 \\
& =2 \lambda^{2} \operatorname{Re}\left\langle A^{2} x_{n}, x_{n}\right\rangle+o(1) \rightarrow 0
\end{aligned}
$$

as $n \rightarrow \infty$ by (2.3). This completes the proof of part (iii).

For the proof of (iv) consider the necessary and sufficient condition (2.6) for $C(H ; A)=2$. Since $A$ and $A^{-1}$ are both bounded, by taking a subsequence if necessary we may assume that $\lim _{n \rightarrow \infty}\left\|A^{2} x_{n}\right\|=\lambda^{2}$ where $\lambda$ is positive. We now verify (2.3). We have $\mu_{n}=\left\|A^{2} x_{n}\right\|^{\frac{1}{2}}+\lambda$ and, by hypothesis, 


$$
\begin{aligned}
& 0=\lim _{n \rightarrow \infty} P_{\mu_{n}} x_{n}=\lim _{n \rightarrow \infty}\left\{P \lambda^{x} n^{+}\left[\left(\mu_{n}-\lambda\right) A x_{n}+\left(\mu_{n}^{2}-\lambda^{2}\right) x_{n}\right]\right\} \\
& =\lim _{n \rightarrow \infty} P \lambda^{x} n
\end{aligned}
$$

since the term in square brackets converges to zero. To complete the proof of (iv) note first that $\alpha_{n} \rightarrow 0$. Next, since $\mu_{n} \rightarrow \lambda$ and $P_{\lambda} x_{n} \rightarrow 0$,

$$
\begin{aligned}
\alpha\left(\mu_{n}, x_{n}\right) & =\alpha\left(\lambda, x_{n}\right)+o(1) \\
& =2 \operatorname{Re}\left(-\lambda^{2} x_{n}, A^{2} x_{n}\right)+o(1) .
\end{aligned}
$$

It follows that $\operatorname{Re}\left(A^{2} x_{n}, x_{n}\right\rangle \rightarrow 0$ as $n \rightarrow \infty$.

REMARK 2.3. Theorem 2.2 (iv) can be generalized as follows. Note that if $A$ is m-dissipative and if $\varepsilon, \delta$ are positive numbers, then the operators $A_{\varepsilon \delta}=A(I-\varepsilon A)^{-1}+\delta I$ are bounded, have bounded inverses, are $m$-dissipative, and converge to $A$ in the following senses as $\varepsilon, \delta \rightarrow 0^{+}$:

$$
\begin{gathered}
A_{\varepsilon \delta} x \rightarrow A x \text { for } x \in D(A), \\
\left(\lambda I-A_{\varepsilon \delta}\right)^{-1} x \rightarrow(\lambda I-A)^{-1} x \text { for } x \in H \text { and } \lambda>0, \\
\exp \left(t A_{\varepsilon \delta}\right) x \rightarrow \exp (t A) x \text { for } x \in H, t \geq 0 .
\end{gathered}
$$

Thus if $C\left(H ; A_{\varepsilon \delta}\right)=2$ for sufficiently small $E$ and $\delta$, we can apply (iv) to $A_{\varepsilon \delta}$ and then use a Cantor diagonalization argument to conclude that (2.3) is a necessary condition for $A$.

REMARK 2.4. For $A=L-I$ with $L$ a contraction, the extremal conditions (2.1) and (2.2) become

$$
\begin{aligned}
L^{2} x+(\lambda-2) L x+\left(\lambda^{2}-\lambda+1\right) x & =0, \\
\operatorname{Re}\left(\left(2 L-L^{2}\right) x, x\right) & =1 .
\end{aligned}
$$

Similar expressions hold in the approximate extremal case.

REMARK 2.5. By Proposition 2.1 and its proof, for $A$ nonzero, m-dissipative and normal on $H, C(H ; A)=1$ and there is an extremal for $A$ if and only if there is a unit vector $x$ and a positive number $\lambda$ such 
that $A^{*} A x=\lambda x$; that is, $A$ has an extremal if and only if $A^{*} A$ has a nonzero eigenvalue. When $A=L-I$ where $L$ is unitary, the equation $A^{*} A x=\lambda x$ becomes, using $L^{*}=L^{-1}$,

$$
L^{2} x+(\lambda-2) L x+x=(L-\alpha I)(L-\beta I) x=0 .
$$

Thus $A$ has an extremal if and only if $L$ has an eigenvalue other than one.

REMARK 2.6. Consider the extremal equation (2.1) to be solved for $\lambda>0$ and $x \in D\left(A^{2}\right)$ when $H$ is complex. Factor this equation as

$$
(A-\alpha I)(A-\beta I) x=0 \text {. }
$$

If $(A-B I) x=0$, then $A x=B x$, whence

$$
\|A x\|^{2}=|B|^{2}\|x\|^{2}=\left\|A^{2} x\right\|\|x\| .
$$

This cannot give $C(H ; A)>1$. It follows that if $x$ is an extremal for $A$ with $C(H ; A)=2$ we must have $y=A x-\beta x \neq 0$ and $A y=\alpha y$. A similar remark holds for approximate extremal sequences.

\section{Series inequalities}

Let $\mathbb{K}$ denote the (real or complex) scalar field. Let $\alpha=-\infty$ or $\alpha=0$ and set

$$
i^{p}(\alpha)=\left\{x=\left\{x_{j}\right\}_{j=\alpha}^{\infty}: x_{j} \in \mathbb{K},\|x\|_{p}=\left(\sum_{j=\alpha}^{\infty}\left|x_{j}\right|^{p}\right)^{1 / p}<\infty\right\}
$$

for $1 \leq p<\infty$ with the usual modification for $p=\infty$. These are, of course, the standard Lebesgue sequence spaces.

THEOREM 3.1 (Copson [2] - note the error in the conclusion of this theorem on page 109). Let $\left\{x_{j}\right\}_{j=-\infty}^{\infty}$ be a sequence of real or complex numbers such that $\sum_{j=-\infty}^{\infty}\left|x_{j}\right|^{2}$ is convergent. Then, for $\Delta x_{j}=x_{j+1}-x_{j}$, $\sum_{j=-\infty}^{\infty}\left|\Delta^{2} x_{j}\right|^{2}$ is convergent and 


$$
\left(\sum_{j=-\infty}^{\infty}\left|\Delta x_{j}\right|^{2}\right)^{2} \leq\left(\sum_{j=-\infty}^{\infty}\left|\Delta^{2} x_{j}\right|^{2}\right)\left(\sum_{j=-\infty}^{\infty}\left|x_{j}\right|^{2}\right)
$$

Equality holds in (3.1) if and only if $x_{j}=0$ for all $j$. The inequality (3.1) is best possible.

THEOREM 3.2 (Copson [2]). Let $\left\{x_{j}\right\}_{j=0}^{\infty}$ be a sequence of real or complex numbers such that $\sum_{j=0}^{\infty}\left|x_{j}\right|^{2}$ is convergent. Then $\sum_{j=0}^{\infty}\left|\Delta^{2} x_{j}\right|^{2}$ is convergent and

$$
\left(\sum_{j=0}^{\infty}\left|\Delta x_{j}\right|^{2}\right)^{2} \leq 4\left(\sum_{j=0}^{\infty}\left|\Delta^{2} x_{j}\right|^{2}\right)\left(\sum_{j=0}^{\infty}\left|x_{j}\right|^{2}\right)
$$

where $\Delta x_{j}=x_{j+1}-x_{j}$ as before. Equality occurs in (3.2) if and only if $x_{j}=0$ for all $j$. Finally the constant 4 in (3.2) is best possible.

Proofs. These results follow readily from the results of the previous section. To prove Theorem 3.1 let $H=\tau_{2}(-\infty)$. Let $L$ be the bilateral shift defined by $L x=y$ where $y=\left\{y_{j}\right\}_{j=-\infty}^{\infty}$ and $y_{j}=x_{j+1}$ for all $j$. Then $L$ is unitary and $L \neq I$. By Proposition 2.I, $C(H ; L-I)=1$. Since $A x=\left\{\Delta x_{j}\right\}_{j=-\infty}^{\infty},(3.1)$ follows. Since $L$ has no eigenvalues, $A$ has no extremals by Remark 2.5. Theorem 3.1 is now proved.

For the proof of Theorem 3.2, let $H=z_{2}(0)$ and define the unilateral shift $L$ by $L x=y$ where $y=\left\{y_{j}\right\}_{j=0}^{\infty}$ and $y_{j}=x_{j+1}$ for all $j \geq 0$. Then $L$ is a contraction on $H$, whence for $A=L-I$, $C(H ; A) \leq 2$ by Proposition 2.1, proving (3.2). It remains to show that $C(H ; A)=2$ and that $A$ has no extremals.

For the moment assume that $C(H ; A)=2$. Then, by Remark 2.5, there are no extremals for $A$ since $L$ has no eigenvalues.

To show that $C(H ; A)=2$ and that an approximate extremal sequence exlsts, we use Theorem 2.2 ( $i$ ii). The extremal equation (2.7) (and the associated approximate extremal equation) is a second order difference equation whose general solution can easily be found explicitly. Doing so 
leads us to look for an approximate extremal sequence $\left\{x_{n}\right\}_{n=1}^{\infty}$ of the form $x_{n}=\left\{x_{n j}\right\}_{j=0}^{\infty}$ where

$$
x_{n j}=\rho_{n}^{j} \sin \left(\alpha_{j} \rho_{n}+\beta_{j}\right), j \geq 0 .
$$

Elementary, but rather tedious calculations, which we omit, show that if we take $\rho_{n}=1-\varepsilon, \alpha_{j}=3^{\frac{1}{2}} j(1-\varepsilon)^{-1}, B_{j}=-\pi / 3$, and $0<\varepsilon<1$, and if we write the resulting $x_{n}$ as $x_{n}^{(\varepsilon)}$, then the sequence $\left\{x_{n}^{(1 / n)}\right\}$ is an approximate extremal sequence for $A$. The calculation is the one hinted at by Copson [2], and this is the one part of Copson's paper that we have been unable to simplify. The proof of Theorem 3.2 is now complete.

\section{Inequalities for m-dissipative operators}

For $A$ an m-dissipative operator on a Banach space $X$ let

$$
C(A, x)=\|A x\|^{2} /\left(\left\|A^{2} x\right\|\|x\|\right)
$$

for $x \in D\left(A^{2}\right)$ with $A^{2} x \neq 0$, so that $C(A, x)$ is the smallest constant $k$ which makes the inequality

$$
\|A x\|^{2} \leq k\left\|A^{2} x\right\|\|x\|
$$

valid. (Consequently $C(X ; A)=\sup _{x} C(A, x)$.) In this section we shall establish some results about $C(A, x)$, especially when $x$ is an $L^{p}$ space. These results complement and improve some of our earlier results [3] and some of those in [4]. Examples include the case when $X=Z^{p}(0)$ and $A$ is the difference operator as in the proof of Theorem 3.2 .

For our first result we use Holbrook's measure $a(X)$ of how "Euclidean" a Banach space $X$ is [4]. Set

$$
a(x)=\sup _{x, y \neq 0} \frac{\|x+y\|^{2}+\|x-y\|^{2}}{2\left(\|x\|^{2}+\|y\|^{2}\right)} .
$$

It is easy to see that $1 \leq a(X) \leq 2$ and that $X$ is a Hilbert space if and only if $a(X)=1$. One interprets $a(X)$ as a measure of how close $X$ 
is to a Hilbert space. Using Clarkson's inequalities [1], Holbrook [4] showed that

$$
a(x) \leq 2^{|1-2 / p|}
$$

if $X$ is a subspace of an $L^{p}$ space.

THEOREM 4.1. For $\lambda>0$ let $B_{\lambda}=(\lambda I+A)(\lambda I-A)^{-1}$ be the cayley transform of an m-dissipative operator $A$ on $X$. Let $M=\sup _{\lambda}\left\{\left\|B_{\lambda}\right\|: \lambda>0\right\}$. Then for alz $x \in O\left(A^{2}\right)$,

$$
\|A x\|^{2} \leq a(x)\left(1+M^{2}\right)\left\|A^{2} x\right\|\|x\| .
$$

Proof. For $x \in D\left(A^{2}\right)$ and $\lambda>0$ we have the identity

$$
2 \lambda A x=\left(A^{2} x+\lambda^{2} x\right)+B_{\lambda}\left(A^{2} x-\lambda^{2} x\right),
$$

from which it follows that

$$
2 \lambda\|A x\| \leq\left\|A^{2} x+\lambda^{2} x\right\|+\|B \lambda\|\left\|A^{2} x-\lambda^{2} x\right\|
$$

The Cauchy inequality $\left(\sum_{j=1}^{2} a_{j} b_{j}\right)^{2} \leq\left(a_{1}^{2}+a_{2}^{2}\right)\left(b_{1}^{2}+b_{2}^{2}\right)$ implies

$$
\begin{aligned}
4 \lambda^{2}\|A x\|^{2} & \leq\left(1+\left\|B_{\lambda}\right\|^{2}\right)\left(\left\|A^{2} x+\lambda^{2} x\right\|^{2}+\left\|A^{2} x-\lambda^{2} x\right\|^{2}\right) \\
& \leq 2 a(x)\left(1+\|B\|^{2}\right)\left(\left\|A^{2} x\right\|^{2}+\lambda^{4}\|x\|^{2}\right)
\end{aligned}
$$

by (4.1). If $A^{2} x \neq 0$, setting $\lambda^{2}=\left\|A^{2} x\right\|\|x\|^{-1}$ yields

$$
\|A x\|^{2} \leq a(X)\left(1+\|B\|^{2}\right)\left\|A^{2} x\right\|\|x\|
$$

and (4.3) follows.

REMARK 4.2. When $X$ is a Hilbert space the above result reduces to Kato's theorem [6] because $a(X)=M=1$ in this case. Also, (4.5) (which gives an estimate on $C(A, x))$ generalizes Theorem 2.2 in [3] because, in the notation of $[3], 1+\left\|B_{\lambda}\right\|^{2} \leq 2 M\left(x ; \lambda_{x}\right)$ and (4.2) holds. Moreover, (4.5) generalizes Theorem 9 of [4] since one can readily check that, in the notation of [4], $\left\|B_{\lambda}\right\| \leq b(x)$. 
THEOREM 4.3. Let $A$ be m-dissipative on $X$, a subspace of an $L^{p}$ space, and let $q$ be the conjugate exponent, $p^{-1}+q^{-1}=1$. Let $x \in \mathcal{D}\left(A^{2}\right)$ with $a^{2} x \neq 0$ and let $\lambda=\lambda_{x}=\left(\left\|A^{2} x\right\| /\|x\|\right)^{\frac{1}{2}}$. If $2 \leq p<\infty$,

$$
\|A x\|^{2} \leq\left(1+\left\|B_{\lambda}\right\|^{q}\right)^{2 / q}\left\|A^{2} x\right\|\|x\|,
$$

while if $1<p \leq 2$,

$$
\|A x\|^{2} \leq\left(1+\left\|B_{\lambda}\right\|^{p}\right)^{2 / p}\left\|A^{2} x\right\|\|x\| .
$$

Proof. Apply Hölder's inequality

$$
\sum_{j=1}^{2} a_{j} b_{j} \leq\left(\left|a_{1}\right|^{p}+\left|a_{2}\right|^{p}\right)^{1 / p}\left(\left|b_{1}\right|^{q}+\left|b_{2}\right|^{q}\right)^{1 / q}
$$

to $(4.4)$ to obtain

$$
\begin{aligned}
2 \lambda\|A x\| & \leq\left(1+\left\|B_{\lambda}\right\|^{q}\right)^{1 / q}\left(\left\|A^{2} x+\lambda^{2} x\right\|^{p}+\left\|A^{2} x-\lambda^{2} x\right\|^{p}\right)^{1 / p} . \\
& \leq\left(1+\left\|B_{\lambda}\right\|^{q}\right)^{1 / q}\left(2^{p-1}\left(\left\|A^{2} x\right\|^{p}+\left\|\lambda^{2} x\right\|^{p}\right)\right)^{1 / p}
\end{aligned}
$$

by one of Clarkson's inequalities $\left[1\right.$, p. 400]. Take $\lambda=\left(\left\|A^{2} x\right\| /\|x\|\right)^{\frac{1}{2}}$, plug in, manipulate and square; then (4.6) comes out. To prove (4.7), one proceeds in a similar manner; only this time the relevant Clarkson inequality $[1, \mathrm{p} .400]$ is

$$
\|x+y\|_{p}^{p}+\|x-y\|_{p}^{p} \leq 2\left(\|x\|_{p}^{p}+\|y\|_{p}^{p}\right)
$$

for $x, y \in L^{p}$ and $1<p \leq 2$.

REMARK 4.4. If $A$ is m-dissipative on a subspace $X$ of $L^{p}$ then a variant of the proof of Theorem 4.1 shows that

$$
\|A x\|^{2} \leq 2^{2-2 / p}\left(c_{1}+c_{2}\right)^{2}\left\|A^{2} x\right\|\|x\|
$$

for $x \in D\left(A^{2}\right)$ with $A^{2} x \neq 0$. Here

$$
c_{1}=\left\|\left(I+B_{\lambda}\right) / 2\right\|, \quad c_{2}=\left\|\left(I-B_{\lambda}\right) / 2\right\|,
$$


and $\lambda=\left(\left\|A^{2} x\right\| /\|x\|\right)^{\frac{1}{2}}$. In the "Copson case" of $A=L-I$ with $L$ a contraction, one easily shows that $c_{1} \leq 1$ and $c_{2} \leq 2$. In some cases these estimates can be improved for certain values of $\lambda$. For instance, $c_{2}=\left\|A(\lambda I-A)^{-1}\right\| \leq\|A\| / \lambda \rightarrow 0$ as $\lambda \rightarrow \infty$. Also, $\quad c_{1}=\left\|\lambda((\lambda+I) I-L)^{-1}\right\| \rightarrow 0$ as $\lambda \rightarrow 0$ if one is in the resolvent set of $L$, that is if $L-I$ has a bounded inverse. If $L$ is dissipative, then

$$
c_{1}=\left\|\lambda((\lambda+1) I-L)^{-1}\right\| \leq \lambda /(\lambda+1)
$$

and from $\left(I+B_{\lambda}\right)+\left(I-B_{\lambda}\right)=2 I$ the inequalities

$$
c_{1} \leq c_{2}+1, c_{2} \leq c_{1}+1
$$

follow.

As in [3], we write $T \in M_{c}$ if $T=\{T(t): t \geq 0\}$ defines a $\left(C_{0}\right)$ contraction semigroup on (the simple functions of) ${ }_{L}{ }^{p}(\Omega, \Sigma, \mu$ ) for each $p, 1<p<\infty$. Let $A$ (or $A_{p}$ ) denote the generator of $T$ acting on $L^{p}$, and let

$$
M_{p}=\sup \left\{\left\|B_{\lambda}\right\|: \lambda>0\right\}
$$

where $B_{\lambda}$ is the Cayley transform of $A_{p}$. Set

$$
M_{1}=\underset{p \rightarrow 1}{\lim \inf } M_{p}, M_{\infty}=\underset{p \rightarrow \infty}{\lim \sup } M_{p} .
$$

THEOREM 4.5. Let $A$ generate $T \in M_{c}$ and let $M_{1}, M_{\infty}$ be as above. Then

$$
C\left(L^{p} ; A\right) \leq 2^{1-2 / P}\left(I+M_{\infty}^{2-4 / p}\right) \leq 2^{1-2 / P}\left(1+3^{2-4 / p}\right)
$$

if $2 \leq p \leq \infty$, while if $1<p \leq 2$,

$$
C\left(L^{p} ; A\right) \leq 2^{2 / p-1}\left(1+M_{1}^{4 / p-2}\right) \leq 2^{2 / p-1}\left(1+3^{4 / p-2}\right) .
$$

Proof. This is proved just like Theorem 2.4 in [3] except that Theorem 4.1 above is used in place of Theorem 2.2 of [3].

REMARK 4.6. Note that, in the above theorem, $C\left(L^{p} ; A\right)<4$ whenever 


$$
1.485 \approx p_{*}<p<p^{*} \approx 3.064
$$

where $p^{*}$ is the unique solution of the transcendental equation

$$
\log _{2} r=\log _{3}((16 r-1) / 9), r=2^{2-2 / p^{*}},
$$

and a similar result holds for $p_{*}$. (The numbers $p^{*}$ and $p_{*}$ were computed approximately on an HP-25 pocket calculator.) of course, $p_{*}^{-1}+p^{*-1}=1$. Compare $(4.8)-(4.10)$ with the poorer estimates (2.11), (2.12) of [3].

Theorem 4.5 can be sharpened as follows. Let $C(A, x)$ be as in the first paragraph of this section.

THEOREM 4.7. Let the hypotheses of Theorem 4.5 hold, and let $1<p<\infty$. Let $\tilde{p}=1$ or $\tilde{p}=\infty$ according as $p \leq 2$ or $p>2$. Let $x \in n\left\{D\left(A_{p}^{2}\right): p\right.$ between 2 and $\left.\tilde{p}\right\}$ and let

$$
\lambda=\inf \left\{\left(\left\|A_{p} x\right\|_{p}^{2} /\|x\|_{p}\right)^{\frac{3}{2}}: p \text { between } 2 \text { and } \tilde{p}\right\} \text {. }
$$

Then

$$
C\left(A_{p}, x\right) \leq 2^{|1-2 / p|}(1+s|1-2 / p|)
$$

where $s=1$ if $\lambda \geq 1$ while $s=((3-\lambda) /(1+\lambda))^{2}$ if $0<\lambda<1$.

Replacing $s$ by $M_{1}^{2}$, or $M_{\infty}^{2}$ gives the estimates (4.8) and (4.9). The theorem is proved by the proof technique of Theorem 4.5; we omit the details.

\section{References}

[1] James A. Clarkson, "Uniformly convex spaces", Trans. Amer. Math. Soc. 40 (1936), 396-414.

[2] E.T. Copson, "Two series inequalities", Proc. Roy. Soc. Edinburgh Sect. A 83 (1979), 109-114. 
[3] Herbert A. Gindler and Jerome A. Goldstein, "Dissipative operator versions of some classical inequalities", J. Analyse Math. 28 (1975), 213-238.

[4] John A.R. Holbrook, "A Kallman-Rota inequality for nearly Euclidean spaces", Adv. in Math. 14 (1974), 335-345.

[5] Robert A. Kallman and Gian-Carlo Rota, "On the inequality $\left\|f^{\prime}\right\|^{2} \leq 4\|f\| \cdot\left\|f^{\prime \prime}\right\| "$, Inequalities, II, 187-192 (Proc. Second Sympos., US Air Force Acad., Colorado, 1967. Academic Press,. New York, 1970).

[6] Tosio Kato, "On an inequality of Hardy, Littlewood, and Pólya", Adv. in Math. 7 (1971), 217-218.

[7] Man Kam Kwong and A. Zett1, "Landau's inequality", submitted.

[8] Man Kam Kwong and A. Zet†I, "Ramifications of Landau's inequality", Proc. Roy. Soc. Edinburgh Sect. A 86 (1980), 175-212.

[9] ю.И. Лобич [Ju.l. L.jubič], "О неравенствах между степенямн линейного one-paeopa" [On inequalities between the powers of a linear operator], Izv. Akad. Nauk SSSR Ser. Mat. 24 (1960), 825-864; English transl: Transl. Amer. Math. Soc. (2) 40 (1964), 39-84.

[10] G. Lumer and R.S. Phillips, "Dissipative operators in a Banach space", Pacific J. Math. 11 (1961), 679-698.

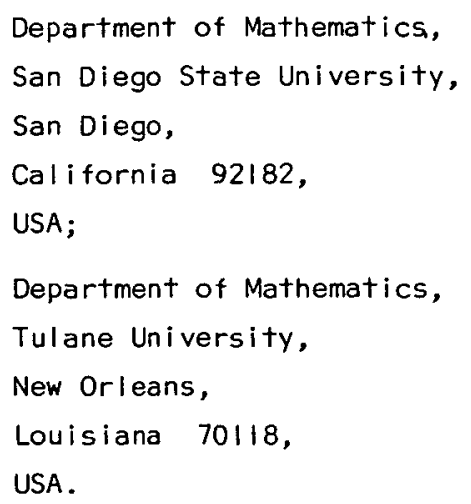

